\title{
Preliminary Results of Solar Flare Induced D-Region Perturbations over UKM Using Stanford AWESOME Receiver
}

\author{
Mohd Masri Abd Rashid1*, Mahamod Ismail1,2, Zamri Zainal Abidin ${ }^{3}$ \\ ${ }^{1}$ Department of Electrical, Electronic and Systems Engineering, Faculty of Engineering and Built Environment, \\ National University of Malaysia, Selangor, Malaysia \\ ${ }^{2}$ Institute of Space Science (ANGKASA), National University of Malaysia, Selangor, Malaysia \\ ${ }^{3}$ Radio Cosmology Research Lab Physics Department, Science Faculty, University of Malaya, Kuala Lumpur, Malaysia \\ Email: * masri rashid@yahoo.co.uk
}

Received 17 March 2015; accepted 5 May 2015; published 11 May 2015

Copyright (C) 2015 by authors and Scientific Research Publishing Inc.

This work is licensed under the Creative Commons Attribution International License (CC BY). http://creativecommons.org/licenses/by/4.0/

c) (i) Open Access

\begin{abstract}
We present the preliminary results of VLF signal perturbations produced due to solar flare. The data were recorded by the Stanford VLF AWESOME receiver located at National University of Malaysia, Selangor. Two new long distance $(>1000 \mathrm{~km})$ VLF paths, JJI-UKM $(2700 \mathrm{~km})$ and NWC-UKM $(3300 \mathrm{~km})$ were analyzed simultaneously. Data from the GOES satellite were used to determine the onset time and type of each of these flares. Results indicated that all five solar flare events with an X-ray peak flux above $10^{-5} \mathrm{~W} / \mathrm{m}^{2}$ (M-class) were recorded, $37.5 \%$ for $\mathrm{X}$-ray flux greater than $10^{-6} \mathrm{~W} / \mathrm{m}^{2}$ (C-class), while the weakest X-ray flux recorded was $2.6 \times 10^{-7} \mathrm{~W} / \mathrm{m}^{2}$ (B-class) with $0.24 \%$ probing potentiality. We found a strong positive correlation $(0.84)$ between solar flare radiation intensity and the values of amplitude and phase perturbations for both paths. The values of amplitude and phase perturbations time-correlated with solar flare, varied from 0.2 to $5 \mathrm{~dB}$ and 0.15 to 20 degree respectively. These findings are in complete agreement with previous works and demonstrate that the data obtained by the UKM AWESOME observation station will provide additional contribution to the study of ELF/VLF waves phenomena in the ionosphere/magnetosphere, especially at low latitudes region.
\end{abstract}

\section{Keywords}

Solar Flare, VLF Signal Perturbation, X-Ray Flux

${ }^{*}$ Corresponding author.

How to cite this paper: Rashid, M.M.A., Ismail, M. and Abidin, Z.Z. (2015) Preliminary Results of Solar Flare Induced D-Region Perturbations over UKM Using Stanford AWESOME Receiver. Journal of Applied Mathematics and Physics, 3, $455-464$. http://dx.doi.org/10.4236/jamp.2015.35058 


\section{Introduction}

Solar flare is a catastrophic explosion that occurs in the Sun's chromospheres. Most solar flare events are accompanied by Coronal Mass Ejections (CMEs) that emit radiations which consist of huge energy particles. The rapid changes of charged particles due to solar flares affect the earth's atmosphere as well as the propagation of very low frequency (VLF, $3-30 \mathrm{kHz}$ ) waves within the Earth Ionosphere Wave Guide (EIWG), located 60 to $70 \mathrm{~km}$ above the earth surface. VLF radio waves can propagate over very long distances due to its minimum attenuation rate which is typically $2-3 \mathrm{~dB}$ per Megameter [1]. Thus, it could be an efficient tool to measure the D-region ionosphere responses scale proportional to the intensity of flares via remote sensing technique.

During day time condition, the subionospheric propagation is normally reflected by a relatively stable reflecting layer at an altitude of about $70 \mathrm{~km}$. This is due to the presence of Lyman- $\alpha$ radiation $(121 \mathrm{~nm})$ from the sun [2]-[5]. The Lyman- $\alpha$ radiation actively increases electrical conductivity at an altitude in the range of $\sim 70-90$ km by ionizing Nitric Oxide (NO) as the Sun rises. However, during the night, the electron recombination processes overcome the ionization. As a result, the D-region disappears when the reflecting layer moves to an altitude of $\sim 90 \mathrm{~km}$.

Apart from solar flare influences, the reasons for excess ionization in the lower ionosphere are due to X-ray burst from remote objects like magnetar burst [6]-[9] and meteor showers [10]. Therefore, strong fundamental knowledge in VLF wave's properties and characteristics is desired in order to understand various kinds of sudden ionosphoric disturbances (SIDs) such as sudden frequency deviations [11] and sudden phase anomalies [12].

Several works were reported earlier on the relationships between the occurrences of solar flare and the ionization behaviour in the lower ionosphere. Reference [13] has done several observations to monitor the characteristics of the $16 \mathrm{kHz}$ VLF narrowband signal during solar flare events. The main objective was to investigate the effect of solar flare activities in the D-region ionosphere over England. In another effort, [14] conducted a study to show the correlation of a weak solar flares X-ray flux with the delay of the amplitude and phase in the VLF transmissions paths.

The classification of a solar flare is based on its x-ray flux intensity output. They are classified according to the order of magnitude of the peak burst intensity (I) measured at the earth in the 0.1 to $0.8 \mathrm{~nm}$ wavelength band as shown in Table 1. A multiplier is used to indicate the level within each class. For example: M6 $=6 \times 10^{-5}$ $\mathrm{W} / \mathrm{m}^{2}$. As a result of rapid responses of VLF signals to changes of ionization rates at the lower ionosphere during flare events, the VLF amplitude and/or phase were altered and these alterations can be observed and recorded using VLF remote sensing technique. The flare occurrences were justified by the GOES (Geostationary Operational Environmental Satellites) satellites and the data was available from the National Oceanic and Atmospheric Administration, Space Weather Prediction Centre (NOAA, SWPC).

\section{Data and Method}

\subsection{Data}

All the VLF recordings were made at UKM, Selangor. The VLF data presented in this work consist of amplitude and/or phase of both NWC and JJI transmitter recorded by Stanford University, Atmospheric Weather Electromagnetic System for Observation, Modeling and Education (AWESOME) instrument. Two transmitters involved in this observation are a $19.8 \mathrm{kHz}$, NWC transmitter, stationed in North West Cape, Australia (21.820S $114.170 \mathrm{E}$ ) and a $22.2 \mathrm{kHz}$, JJI transmitter located in Ebino, Japan (32.040N 130.810E). Figure 1(a) shows the NWC and JJI transmitter locations, the VLF receiver at UKM, Malaysia and the great circle paths (GCPs). JJIUKM VLF signals travelled over almost totally ocean path while NWC-UKM signal path is part-sea-based and part-land-based, providing two new VLF links to explore occurrence characteristics of VLF signal perturbation

Table 1. The solar flare classifications. (Source: www.spaceweatherlive.com, 2003-2014)

\begin{tabular}{cc}
\hline Class & $\mathrm{W} / \mathrm{m}^{2}(0.1$ to $0.8 \mathrm{~nm}$ band $)$ \\
A & $<10^{-7}$ \\
B & $\geq 10^{-7}$ to $<10^{-6}$ \\
C & $\geq 10^{-6}$ to $<10^{-5}$ \\
M & $\geq 10^{-5}$ to $<10^{-4}$ \\
X & $\geq 10^{-4}$ to $<10^{-3}$ \\
\hline
\end{tabular}




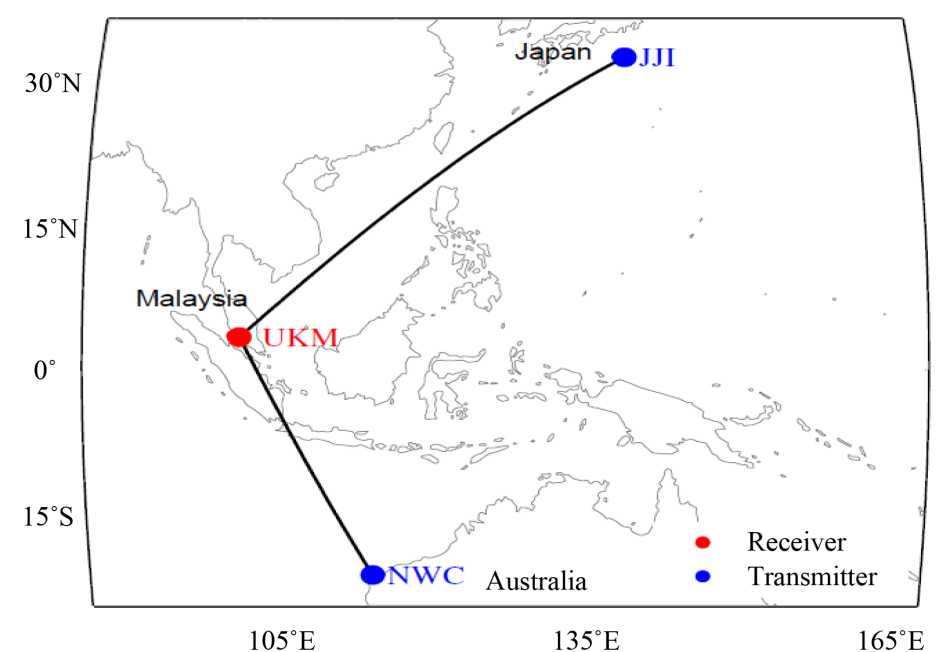

(a)

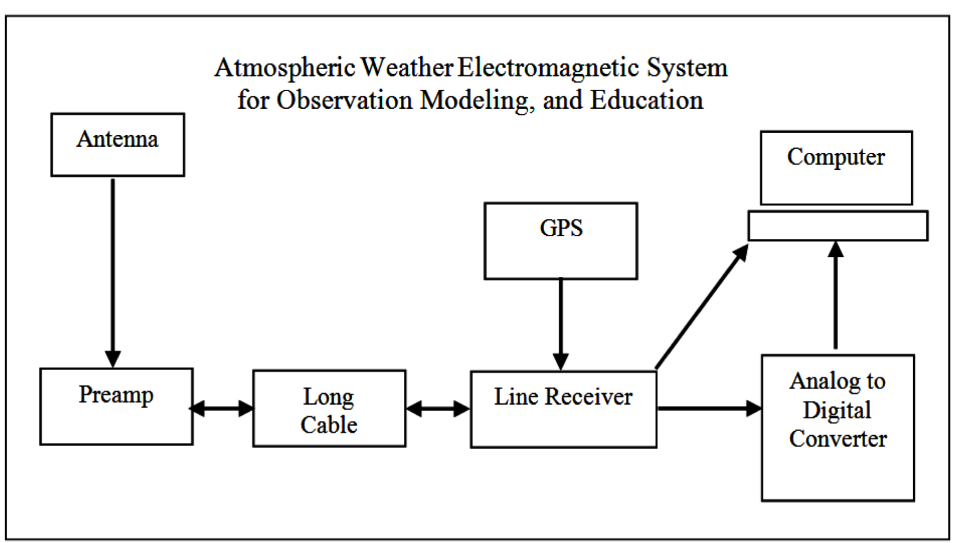

(b)

Figure 1. (a) NWC and JJI transmitter locations, VLF receiver at UKM, Malaysia and the great circle paths (GCPs); (b) AWESOME VLF receiver block diagram. (Source: [20])

events simultaneously. The receiver was installed at UKM (2.550N, 101.460E) in 2009. It was granted by NASA as part of the International Heliophysical Year program (IHY) and the United States Basic Space Science (UNBSS) initiative.

\subsection{Method}

The Stanford VLF receiver observation site involved four main components. These are 1) antennas and cables, 2) preamplifier box, 3) line receiver box and 4) computer. Figure 1(b) shows the block diagram of the AWESOME receiver. The Global Positioning System (GPS) was used to determine very precise date and timing signal so that the data were sampled accurately and synchronized with other VLF receivers' worldwide. In order to have precise timing, the GPS was installed within 60 feet of the main storage computer's location. The VLF antenna is considered the most crucial parts in the AWESOME receiver. The crossed loop antennas were used to detect electromagnetic signals ranging from femtotesla and picotesla with antenna loops aligned in the East-West (E/W) and North-South (N/S) directions.

A preamplifier fixed near the antenna passes the radio signals ranged from $300 \mathrm{~Hz}-47 \mathrm{kHz}$ to the line receiver. The received continuous data is sampled at $100 \mathrm{kHz}$ and is then converted to a digital format by a 16-bit ADC card mounted into the PC. The receiver box should be placed within 1 - 2 meters from the PC to minimize signal attenuation. Data acquisition software provided by Stanford University saves the data in $\mathrm{m}$-file formats. The PC used should at least be a Pentium IV with 512 MB and 80 GB in hard drive space. It must have an open 
PCI slot to install the National Instruments 6043E digitization card. Although it is important that the antenna is installed at a quiet location, in certain circumstances rooftops of a building is also suitable. More comprehensive details of specification, calibration and guide on the installation of the VLF receiver can be found in [15].

\subsection{Determination of Perturbation Parameters}

The physical parameters involved in the estimation of the amplitude perturbations are; 1) $\Delta A=A_{\max }-A_{\text {Min }}$, where $A_{\text {Max }}$ is the full perturbed amplitude increment; and $A_{\text {Min }}$ is the ambient levels (unperturbed values) during no flare condition, 2) T1-rise time, the time taken for the signal to reach its peak value and 3) T2-relaxation time which represent the time the signal returns to its ambient level. The calculation technique was introduced by [16]. In this work, only amplitude perturbations were measured for JJI-UKM path due to JJI transmitter is a phase unstable transmitter as quoted by [17]. Figure 2 illustrate further details on the method used to calculate the above mentioned parameters.

\section{Observational Result}

\subsection{M-Class Flare Events}

During the whole study period, 9 January to 15 December 2010, there were five M-Class flares detected by the UKM receiver ranging from M1.0 to M6.4 flare. The strongest M-Class flare was a $6.4 \times 10^{-5} \mathrm{~W} / \mathrm{m}^{2}$ recorded on 7 February 2010. The NWC-UKM path exhibited an amplitude perturbation $(\Delta \mathrm{A})$ of $\sim 4.0 \mathrm{~dB}$ and $\sim 60$ degree in phase shifting $(\Delta \Phi)$ while JJI-UKM path recorded $\Delta \mathrm{A}=5 \mathrm{~dB}$. However no reading in $\Delta \Phi$ for JJI signal was recorded. The JJI-UKM phase of signal for all events was not stable to be analyzed as stated in previous section.

The M6.4 flare started at 02:22 UT, peaked at 02:32 UT, and returned to its ambient level at 03:03 UT. The rise time, $\mathrm{T} 1$ for the M6.4 flare was $\sim 10$ minutes while the relaxation time, T2 was $\sim 31$ minutes. The other four M-Class flare events were M4.0 flare which occurred on 8 February, M2.0 on 12 June. One M-Class flares on 20th January with the scale of M1.0 plus another M1.0 flares which occurred on 13 June. Five out of six MClass flare events detected by GOES during daytime in Malaysia (UT +8$)$ were successfully recorded by UKM receiver during the study period. These facts proof that the UKM receiver is working in good condition despite several episodes of power and software failures during its early operation in 2009. The full list of flare events properties such as date, time, $\Delta \mathrm{A}, \Delta \Phi, \mathrm{T} 1$ and $\mathrm{T} 2$ recorded in UKM are presented in Table 2.

\subsection{C-Class Flare Event}

There were 32 flare events classified as C-Class flares ranging from C1.1 to C9.9. The highest C-Class flare

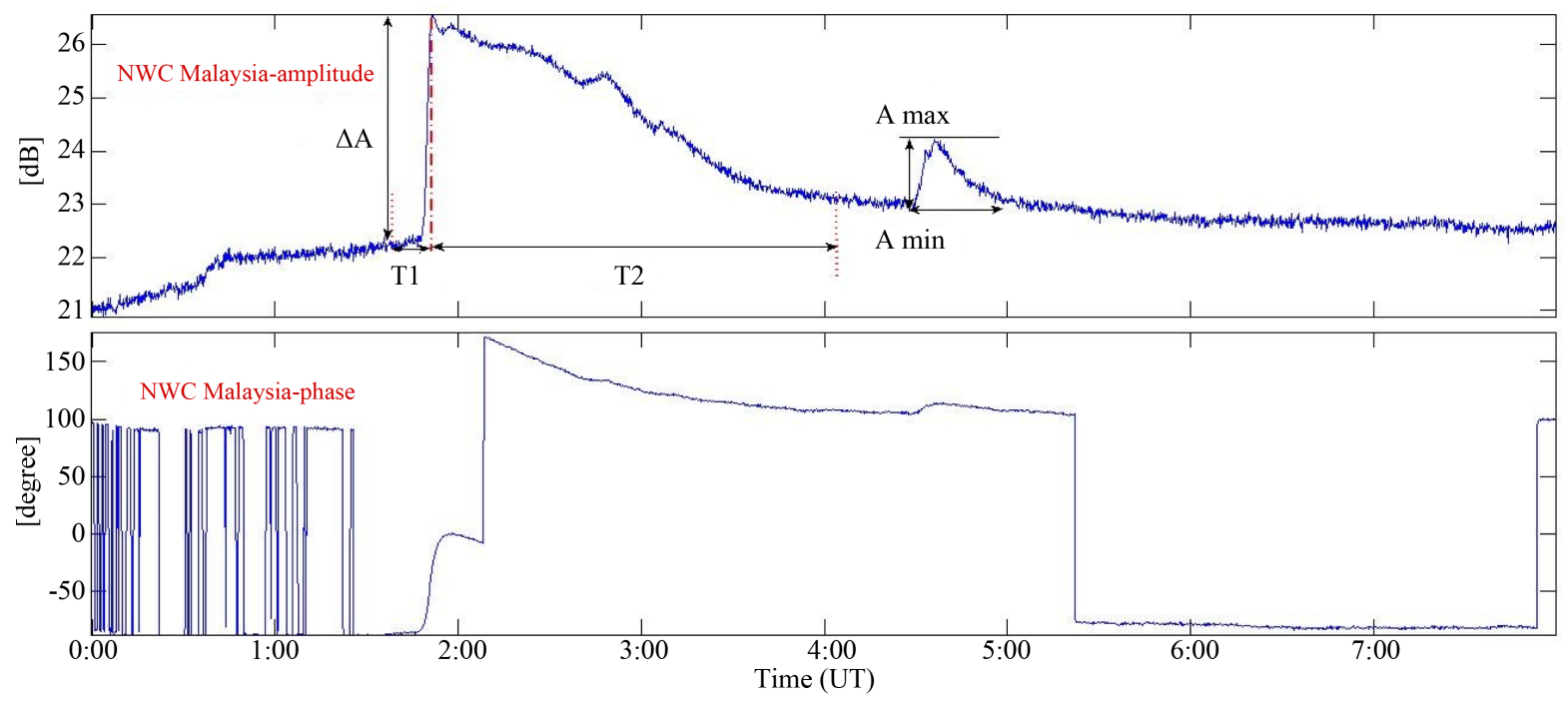

Figure 2. VLF amplitude perturbations and estimation parameters of solar flare; $\Delta \mathrm{A}\left(\mathrm{A}_{\max }-\mathrm{A}_{\mathrm{Min}}\right)$, $\mathrm{T} 1$ and $\mathrm{T} 2$. (Source: [20]) 
Table 2. VLF recorded events observed on the NWC and JJI signals received in UKM, Malaysia.

\begin{tabular}{|c|c|c|c|c|c|c|c|c|c|c|c|c|}
\hline \multirow{2}{*}{ No. } & \multirow{2}{*}{$\begin{array}{l}\text { Date } \\
\text { (2010) }\end{array}$} & \multicolumn{3}{|c|}{ Flare Times (UT) } & \multirow{2}{*}{$\begin{array}{l}\text { Flare } \\
\text { Class }\end{array}$} & \multirow{2}{*}{ Flare Power } & \multicolumn{2}{|c|}{$\Delta \mathrm{A}(\mathrm{dB})$} & \multicolumn{2}{|c|}{$\Delta$ Phase (degree) } & \multirow{2}{*}{$\begin{array}{c}\begin{array}{c}\text { Rise Time } \\
\text { (min) }\end{array} \\
\mathrm{T} 1\end{array}$} & \multirow{2}{*}{$\begin{array}{c}\text { Relaxation } \\
\text { Time (min) } \\
\text { T2 }\end{array}$} \\
\hline & & Start & Max & End & & & JJI & NWC & JJI & NWC & & \\
\hline 1 & 19 Jan & 834 & 845 & 914 & C5.2 & 7.12 & - & 0.7 & - & - & 11 & 29 \\
\hline 2 & 20 Jan & 448 & 457 & 503 & C5.0 & 6.99 & 2.0 & 2.0 & - & 0.15 & 9 & 6 \\
\hline 3 & 20 Jan & 702 & 727 & 735 & M1.0 & 10.0 & 1.8 & 1.8 & - & 0.40 & 25 & 8 \\
\hline 4 & 21 Jan & 119 & 121 & 125 & C4.9 & 6.90 & 3.0 & 1.8 & - & 3.0 & 2 & 4 \\
\hline 5 & 21 Jan & 349 & 354 & 358 & C2.3 & 3.62 & 1.2 & - & - & - & 5 & 4 \\
\hline 6 & 6 Feb & 659 & 704 & 709 & $\mathrm{C} 4.0$ & 6.02 & 1.4 & 1.2 & - & - & 5 & 5 \\
\hline 7 & $7 \mathrm{Feb}$ & 222 & 232 & 303 & M6.4 & 18.1 & 4.5 & 4.0 & - & - & 10 & 31 \\
\hline 8 & 7 Feb & 439 & 452 & 516 & С9.9 & 9.96 & 2.0 & 2.4 & - & - & 13 & 24 \\
\hline 9 & 8 Feb & 315 & 317 & 336 & C6.2 & 7.92 & 2.3 & - & - & - & 2 & 19 \\
\hline 10 & 8 Feb & 355 & 356 & 402 & C2.4 & 3.80 & 0.7 & - & - & - & 1 & 6 \\
\hline 11 & $8 \mathrm{Feb}$ & 411 & 414 & 444 & C7.7 & 8.86 & 3.0 & - & - & - & 3 & 30 \\
\hline 12 & 8 Feb & 516 & 516 & 556 & C8.6 & 9.34 & 1.8 & - & & - & 0 & 40 \\
\hline 13 & 8 Feb & 557 & 607 & 625 & C6.8 & 8.32 & 1.0 & - & - & - & 10 & 18 \\
\hline 14 & 8 Feb & 700 & 701 & 708 & C1.9 & 2.79 & 0.6 & - & - & - & 1 & 7 \\
\hline 15 & 8 Feb & 736 & 743 & 746 & M4.0 & 16.0 & 3.5 & 4.0 & - & - & 7 & 3 \\
\hline 16 & $8 \mathrm{Feb}$ & 803 & 807 & 810 & C1.9 & 2.79 & - & 0.8 & - & - & 4 & 3 \\
\hline 17 & $8 \mathrm{Feb}$ & 954 & 958 & 1003 & C2.8 & 4.47 & 0.7 & 0.6 & - & - & 4 & 5 \\
\hline 18 & 9 Feb & 119 & 123 & 144 & C2.7 & 4.31 & 1.3 & 0.2 & - & - & 4 & 21 \\
\hline 19 & 9 Feb & 412 & 417 & 430 & C2.4 & 3.80 & 1.0 & 0.2 & - & - & 5 & 13 \\
\hline 20 & $12 \mathrm{Feb}$ & 722 & 724 & 737 & C7.9 & 8.97 & 1.8 & 1.8 & - & - & 2 & 13 \\
\hline 21 & $13 \mathrm{Feb}$ & 746 & 752 & 806 & C4.3 & 6.33 & 1.1 & 1.0 & - & - & 6 & 14 \\
\hline 22 & 12 Jun & 055 & 056 & 113 & M2.0 & 13.0 & 3.0 & 2.6 & - & - & 1 & 17 \\
\hline 23 & 12 Jun & 859 & 922 & 940 & C6.1 & 7.85 & 1.7 & 1.0 & - & - & 23 & 18 \\
\hline 24 & 13 Jun & 535 & 535 & 540 & M1.0 & 10.0 & 2.2 & 2.4 & - & - & 0 & 5 \\
\hline 25 & $27 \mathrm{Jul}$ & 412 & 424 & 434 & C2.2 & 3.42 & 0.7 & 0.6 & - & - & 12 & 10 \\
\hline 26 & 18 Aug & 445 & 548 & 651 & C4.5 & 6.53 & - & 2.3 & - & - & 3 & 3 \\
\hline 27 & 26 Sept & 700 & 704 & 712 & B2.6 & -5.85 & - & 1.0 & - & - & 4 & 8 \\
\hline 28 & 31 Oct & 317 & 317 & 328 & C1.8 & 2.55 & 0.8 & 0.5 & - & - & 0 & 11 \\
\hline 29 & 31 Oct & 426 & 431 & 436 & C5.7 & 7.56 & 1.7 & 2.3 & - & 20.0 & 5 & 5 \\
\hline 30 & $3 \mathrm{Nov}$ & 554 & 611 & 626 & C3.8 & 5.80 & - & 1.2 & - & 7.0 & 7 & 15 \\
\hline 31 & $5 \mathrm{Nov}$ & 048 & 056 & 102 & C9.7 & 9.87 & - & 1.3 & - & 6.0 & 8 & 6 \\
\hline 32 & $7 \mathrm{Nov}$ & 137 & 139 & 152 & C5.4 & 7.32 & - & 1.2 & - & - & 2 & 13 \\
\hline 33 & $11 \mathrm{Nov}$ & 213 & 215 & 223 & C2.9 & 4.62 & 1.0 & 0.6 & - & - & 2 & 8 \\
\hline 34 & $11 \mathrm{Nov}$ & 510 & 524 & 539 & C1.1 & 0.41 & - & 0.5 & - & - & 14 & 15 \\
\hline 35 & $11 \mathrm{Nov}$ & 721 & 724 & 734 & C4.7 & 6.72 & 2.5 & 0.4 & - & - & 3 & 10 \\
\hline 36 & $12 \mathrm{Nov}$ & 133 & 134 & 142 & C4.6 & 6.62 & - & 0.6 & - & - & 1 & 8 \\
\hline 37 & $12 \mathrm{Nov}$ & 802 & 810 & 826 & C1.5 & 1.76 & - & 0.8 & - & - & 8 & 16 \\
\hline 38 & 15 Dec & 627 & 639 & 650 & C5.3 & 7.24 & 0.5 & 1.0 & - & - & 12 & 11 \\
\hline
\end{tabular}


event occurred on 7 February, rated at $9.9 \times 10^{-6} \mathrm{~W} / \mathrm{m}^{2}$ with $\Delta \mathrm{A}=2.4 \mathrm{~dB}$. This flare started at 04:39 UT peaked at 04:52 UT, and returned to its pre-flare level at 05:16 UT. The rise time, T1 for the C9.9 flare was 13 minutes and its T2 was $\sim 24$ minutes. The moderate C-Class flare occurred on 31 Oct, rated $5.7 \times 10^{-6} \mathrm{~W} / \mathrm{m}^{2}$ with $\Delta \mathrm{A}=$ $2.3 \mathrm{~dB}$. This flare erupted at 04:26 UT peaked at 04:31 UT and neutralized at 04:36 UT. While the weakest C-Class flare recorded on 11 November, rated at $1.1 \times 10^{-6} \mathrm{~W} / \mathrm{m}^{2}$ with $\Delta \mathrm{A}=0.5 \mathrm{~dB}$ started at 05:10 UT, reached its peak at 05:24 UT and ended at 05:39 UT. The detail properties of all recorded C-Class flare events are as tabulated in Table 2.

\subsection{B-Class Flare Event}

Only one event was recorded for B-Class flare. It was a $2.6 \times 10^{-7} \mathrm{~W} / \mathrm{m}^{2}$ dated 26 September 2010, which erupted at 07:00 UT, peaked at 07:04 and ended at 07:12 UT. The $\triangle \mathrm{A}$ for this event is $\sim 1 \mathrm{~dB}$ (NWC-UKM path). In line with those papers presented earlier, it's quite difficult to detect smaller scale of flare event as compared to a larger one. This is due to the distance of the receiver which is located $\sim 70-90 \mathrm{~km}$ from the lower ionosphere reflecting layer.

\section{Discussion}

VLF stations are part of AWESOME network, operating globally to study electromagnetic waves in ELF and VLF bands. To investigate the influence of flare in the lower ionosphere, we need to characterize the VLF amplitude and/or phase of the VLF recorded data. This is done by estimating the maximum amplitude $\triangle \mathrm{A}$ and phase variation $\Delta \Phi$, as well as other significant parameters related to ELF/VLF phenomenon. The selected VLF data took into account the day and night terminator effect. We consider only the event that occurred between sunrise and sunset of local time (UT + 8). During the whole study period, there were 59 VLF amplitude perturbations due to 38 flare events captured by the VLF AWESOME receiver. Of these 38 flares, 5 are classified as M-Class flare, 32 of $C$ classes and 1 of B class. We removed those events for which the cause is unlikely to be due to the solar flare (unstable signals during dusk and dawn transition period) as referred to NOAA, SWPC.

All the flares studied in this work were recorded when the signal from both NWC and JJI transmitter travelled during the daylight. Figure 3 displays overall monthly distribution of VLF perturbation associated with flare events throughout the year 2010. The month of February 2010 recorded the highest number of ionospheric disturbances with 25 events detected in both TRGCPs. There was no event recorded for the three consecutive months of March, April and May due to software failure and maintenance. However, there were two M-Class events captured in June, followed by several number of C-Class flares including the weakest B-Class flare event occurred between July to December 2010 to prove that the system was recovering.

Figure 4 indicates the observational result on the relationship between $\Delta \mathrm{A}$ and solar X-ray flux intensity for both VLF paths. It is seen from Figure 4(a) that, the scatter plot for JJI-UKM consists of two parts: for the low-

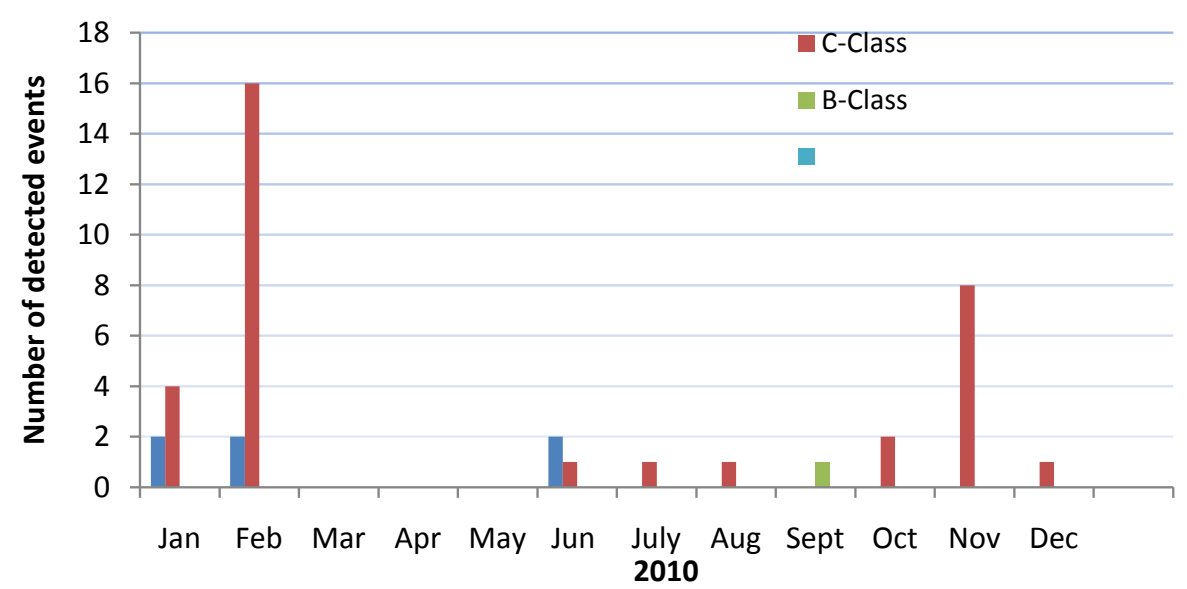

Figure 3. Distribution chart shows flare events recorded by UKM VLF receiver in 2010. The red bar represents C-Class flare. The blue bar represents M-Class while the green represents B-Class flares. 


\section{JJI - UKM path}

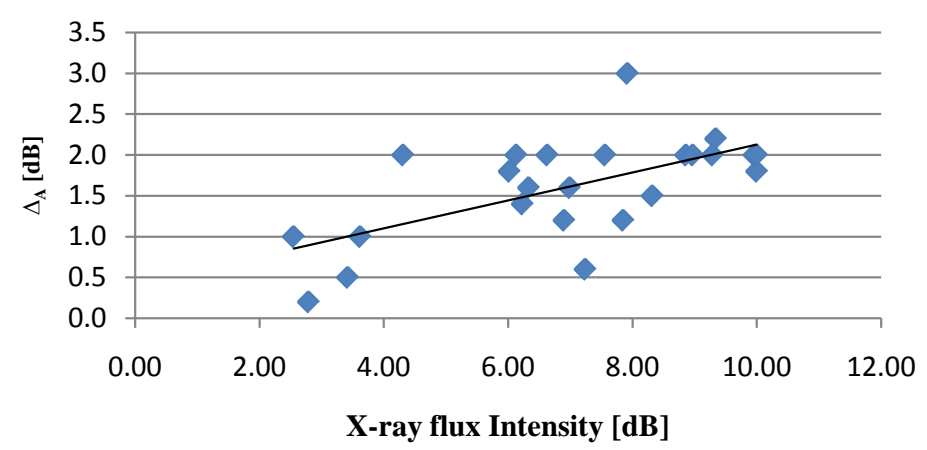

(a)

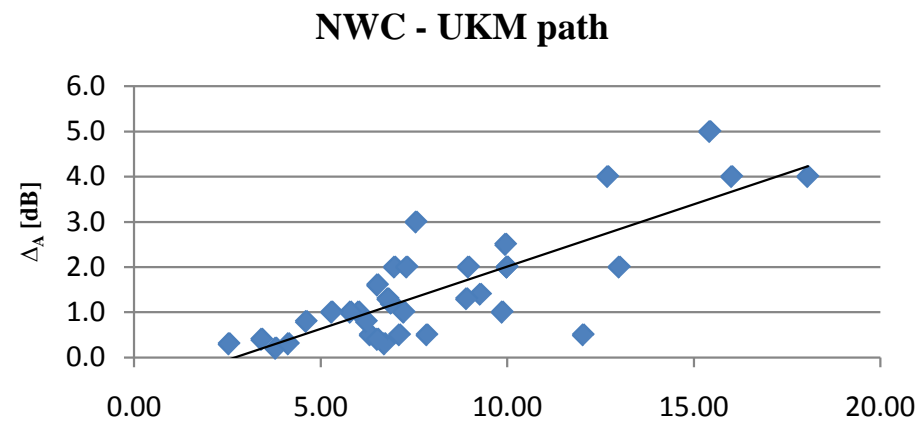

X-ray flux Intensity [dB]

(b)

Figure 4. (a) Scatter plot of observational facts of $\Delta \mathrm{A}$ versus $\mathrm{X}$-ray flux for JJI-UKM path; (b) Equivalent plot for NWC-UKM path.

er part the scatter plot of $\Delta \mathrm{A}$ is drastically increased with the X-ray flux intensity between $\sim 2$ to $10 \mathrm{~dB}$. Taking into account the linear correlation between these two quantities, the cross-correlation coefficient value is 0.622 (medium correlation). Somehow for the higher $\mathrm{X}$-ray flux, the maximum $\Delta \mathrm{A}$ is $\sim 3 \mathrm{~dB}$ with cross-correlation value of -0.04 (no correlation). This is almost similar to and consistent with previous work done by [14] and [16]. Their $\Delta \mathrm{A}$ for the higher $\mathrm{X}$-ray flux saturated at $\sim 4 \mathrm{~dB}$ and $\sim 3 \mathrm{~dB}$ respectively. In our case, the saturated value of $\triangle \mathrm{A}$ was $\sim 3 \mathrm{~dB}$.

Moving on to Figure 4(b) which represents the NWC-UKM path, it seems to us that the plot is considerably more scattered compared to the JJI-UKM path. The $\Delta \mathrm{A}$ increases with the X-ray flux intensity in a range of up to $10 \mathrm{~dB}$. Once the X-ray flux intensities were above $12 \mathrm{~dB}, \Delta \mathrm{A}$ seems to be constant or saturated (at $\sim 5 \mathrm{~dB}$ ). The cross-correlation coefficient is found to be 0.80 which is sufficiently high. Thus, this indicates a strong correlation between $\triangle \mathrm{A}$ and $\mathrm{X}$-ray flux intensity although there is a significant time delay due to the sluggishness of the ionosphere.

It has been observed that all solar flare-associated VLF events (Table 2 and Figure 4) were followed by positive polarity amplitude and phase perturbations. The results in Figure 4 suggest that amplitude and phase perturbations versus the flare power intensity displayed almost linear variations in accordance with [18] [19]. The result obtained from UKM receiver and the GOES satellite was in agreement with typical ionospheric parameters during perturbed condition and illustrate the system's capability in measuring ionospheric properties. Therefore, we can conclude that the VLF remote sensing technique can effectively be utilized to detect the ionospheric electron density enhancements associated with solar flare events.

Unlike most of the previous studies which focused only on a single event or a particular strength of solar flare events (Dahlgren et al. 2011) and etc., we analyzed both large and small scale events from X-ray peak flux greater than $1.0 \times 10^{-7} \mathrm{~W} / \mathrm{m}^{2}$ (B-Class) to an X-ray peak flux greater than $1.0 \times 10^{-5} \mathrm{~W} / \mathrm{m}^{2}$ (M-Class) for the whole year of 2010. As a result, we present the summary of the plotted results of significant X-ray flux events 
ranging from B-Class to M-class flares corresponding to GOES 0.1 to $0.8 \mathrm{~nm}$ wavelength band as shown in Figures 5(a)-(i). The VLF signal profiles presented in Figure 5 clearly demonstrates that during solar flares, there is an abrupt increase in X-ray flux intensity which leads to an increase ionization rate and electron density which modifies the amplitude and/or phase of VLF signals. This result is extremely similar to the hypothesis that the unperturbed D-region layer which reflects the VLF signals is maintained mainly by Lyman- $\alpha$ radiation (121 nm).

\section{Conclusion}

The potentiality of the UKM VLF Stanford AWESOME receiver in probing the solar flare events has been presented. Results shown in Table 2 and Figure 5 imply that the UKM AWESOME receiver is suitable to monitor the overall variations of amplitude/phase perturbation during flare events. The maximum enhancement of amplitude perturbation of up to $5 \mathrm{~dB}$ compared to quiet day have been observed during solar minimum period of 2010. Further analysis revealed that most of the VLF disturbed signal generated by the intense flare X-ray fluxes possessed longer recovery durations as compared to their moderate counterparts. In addition, the system has recorded a weaker flare as compared to the South American VLF Network (SAVNET) receiver. The high value of cross-correlation coefficient obtained from Figure 4 revealed that the magnitude of $\Delta \mathrm{A}$ was directly proportional to the X-ray flux intensity of the solar flares. Although data point for the phase perturbation $(\Delta \Phi)$ versus
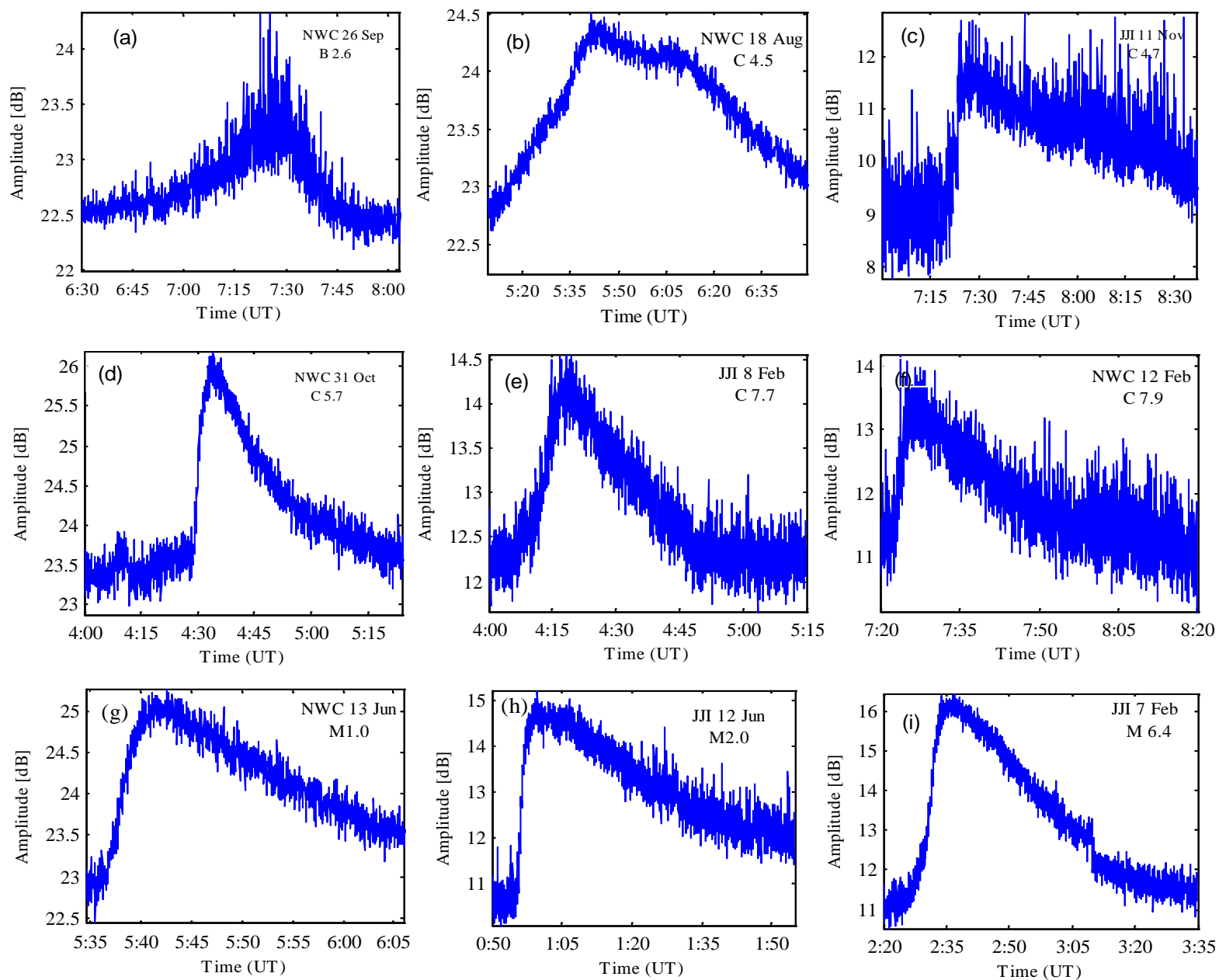

Figure 5. Sample cases of solar flare events ranging from B to M-Class corresponding to GOES 0.1 to 0.8 nm wavelength band recorded at UKM. The date and the GOES Class are indicated and the perturbed signals are as follows: (a) $\mathrm{B} 2.6(\triangle \mathrm{A}=$ $\sim 1 \mathrm{~dB})$; (b) $\mathrm{C} 4.5(\Delta \mathrm{A}=\sim 2.0 \mathrm{~dB})$; (c) $\mathrm{C} 4.7(\Delta \mathrm{A}=\sim 3.0 \mathrm{~dB})$; (d) $\mathrm{C} 5.7(\Delta \mathrm{A}=\sim 2.5 \mathrm{~dB})$; (e) $\mathrm{C} 7.7(\Delta \mathrm{A}=\sim 3.0 \mathrm{~dB}) ;(\mathrm{f}) \mathrm{C} 7.9(\Delta \mathrm{A}$ $=\sim 1.8 \mathrm{~dB})$; $(\mathrm{g}) \mathrm{M} 1.0$ flare $(\Delta \mathrm{A}=\sim 2.4 \mathrm{~dB})$; (h) M2.0 flare $(\Delta \mathrm{A}=\sim 5 \mathrm{~dB})$; and (i) M6.4 flare $(\Delta \mathrm{A}=\sim 4.2 \mathrm{~dB})$. 
X-ray flux intensity could not be plotted due to unstable phase signals received from JJI transmitter, we found that most of the results obtained were in agreement with those written by previous authors.

\section{Acknowledgements}

This work was supported by the research grant DPP-2013-006. The authors would like to express their gratitude to Stanford University VLF research group leads by Prof. Umran Inan for their continuous encouragement and support. The authors would also like to thank Majlis Amanah Rakyat (MARA) for their financial support throughout the research.

\section{References}

[1] Swanson, E.R. (1983) Omega. Proceedings of the IEEE, 71, 1140-1155. http://dx.doi.org/10.1109/PROC.1983.12743

[2] Nicolet, M. and Aikin, A.C. (1960) The Formation of the D Region of the Ionosphere. Journal of Geophysical Research, 65, 1469-1483. http://dx.doi.org/10.1029/JZ065i005p01469

[3] Thomson, N.R. (1993) D-Region Electron Distributions in Middle Latitudes Deduced from the Reflection of Long Radio Waves. Proceedings of the Royal Society of London. Series A, Mathematical and Physical Sciences, 291, 413-437.

[4] Thomson, N.R. (1993) Experimental Daytime VLF Ionospheric Parameters. Journal of Atmospheric and Solar-Terrestrial Physics, 55, 173-184. http://dx.doi.org/10.1016/0021-9169(93)90122-F

[5] McRae, W.M. and Thomson, N.R. (2000) VLF Phase and Amplitude: Daytime Ionospheric Parameters. Journal of Atmospheric and Solar-Terrestrial Physics, 62, 609-618. http://dx.doi.org/10.1016/S1364-6826(00)00027-4

[6] Raulin, J.P., Bertoni, F.C.P. and Rivero, H. (2009) The South America VLF 331 Network (SAVNET). IAU 27th General Assembly, Rio de Janeiro, August 2009, 383.

[7] Kaufmann, P., Piazza, L.R. and Ananthakrishnan, S. (1973) Possible Low Ionosphere Response to Very Hard X-Rays from Cygnus X-3 Bursts in September 1972. Astrophysics and Space Science, 22, 67-70. http://dx.doi.org/10.1007/BF00642822

[8] Rizzo Piazza, L., Kaufmann, P. and Ramirez Pardo, P. (1983) VLF Ionosonde and Long-Distance Propagation Anomalies Produced by Galactic Cen X-4 X-Ray Burst in May 1979. Journal of Atmospheric and Solar-Terrestrial Physics, 45, 121-125. http://dx.doi.org/10.1016/S0021-9169(83)80016-6

[9] Tanaka, Y.T., Terasawa, T., Yoshida, M., Horie, T. and Hayakawa, M. (2008) Ionospheric Disturbances Caused by SGR 1900 + 14 Giant Gamma Ray Flare in 1998: Constraints on the Energy Spectrum of the Flare. Journal of Geophysical Research, 113, A07307. http://dx.doi.org/10.1029/2008JA013119

[10] Chilton, C.J. (1961) VLF Phase Perturbation Associated with Meteor Shower Ionization. Journal of Geophysical Research, 66, 379-383. http://dx.doi.org/10.1029/JZ066i002p00379

[11] Liu, J.Y., Chiu, C.S. and Lin, C.H. (1996) The Solar Flare Radiation Responsible for Sudden Frequency Deviation and Geomagnetic Fluctuation. Journal of Geophysical Research, 101, 10, 855.

[12] Ohshio, M. (1971) Negative Sudden Phase Anomaly. Nature Physics SCI, 229, 239-240. http://dx.doi.org/10.1038/physci229239a0

[13] Deeks, D.G. (1966) D-Region Electron Distributions in Middle Latitudes Deduced from the Reflection of Long Radio Waves. Proceedings of the Royal Society of London. Series A, Mathematical and Physical Sciences, 291, 413-437. http://dx.doi.org/10.1098/rspa.1966.0103

[14] McRae, W.M. and Thomson, N.R. (2004) Solar Flare Induced Ionospheric D-Region Enhancements from VLF Phase and Amplitude Observations. Journal of Atmospheric and Solar-Terrestrial Physics, 66, 77-87. http://dx.doi.org/10.1016/j.jastp.2003.09.009

[15] Cohen, M.B., Inan, U.S. and Paschal, E.W. (1971) Sensitive Broadband ELF/VLF Radio Reception with the Awesome Instrument. IEEE Transactions on Geoscience and Remote Sensing, 48, 3-17. http://dx.doi.org/10.1109/TGRS.2009.2028334

[16] Todoroki, Y., Maekawa, S., Yamauchi, T., Horie, T. and Hayakawa, M. (2007) Solar Flare Induced D-Region Perturbation in the Ionosphere, as Revealed from a Short Distance VLF Propagation Path. Geophysical Research Letters, 34, L03103.

Afraimovich, E.L., Kosogorov, E.A., Leonovich, L.A., Palamartchouk, K.S., Perevalova, N.P. and Pirog, O.M. (2000) Determining Parameters of Large-Scale Traveling Ionospheric Disturbances of Auroral Origin Using GPS-Arrays. Journal of Atmospheric and Solar-Terrestrial Physics, 62, 553-565. http://dx.doi.org/10.1016/S1364-6826(00)00011-0

[17] Maurya, A.K., Singh, R., Veenadhari, B., Kumar, S. and Singh, A.K. (2013) Sub-Ionospheric Very Low Frequency Perturbations Associated with the 12 May 2008 M = 7.9 Wenchuan Earthquake. Natural Hazards and Earth System Sciences, 
13, 2331-2336. www.nat-hazards-earth-syst-sci.net/13/2331/2013/ http://dx.doi.org/10.5194/nhess-13-2331-2013

[18] Dahlgren, H., Sundberg, T., Collier, A.B., Koen, E. and Meyer, S. (2011) Solar Flares Detected by the New Narrowband VLF Receiver at SANAE IV. South African Journal of Science, 107, 8 p. http://dx.doi.org/10.4102/sajs.v107i9/10.491

[19] Koen, E.J. and Collier, A.B. (2013) Mid-Latitude Ionospheric Signature of a Weak Solar Flare in Winter. South African Journal of Science, 109, 1.

[20] Rashid, M.M., Ismail, M., Hasbie, A.M., Salut, M.M. and Abdullah, M. (2013) VLF Observation of D-Region Disturbances Associated with Solar Flares at UKM Selangor Malaysia. Proceeding of the 2013 IEEE International Conference on Space Science and Communication (ICONSPACE), Melaka, July 2013, 249-252. http://dx.doi.org/10.1109/IconSpace.2013.6599474 